\title{
NLO event samples for the LHC
}

\author{
M.V. GARZELLI, Adam KARDOS and Zoltán TRÓCSÁNYI*i \\ Institute of Physics, University of Debrecen, H-4010 Debrecen P.O.Box 105, Hungary \\ E-mail: Z.Trocsanyi@atomki.hu
}

\begin{abstract}
We introduce a twiki page with collections of generated Monte Carlo event samples in protonproton collisions at LHC energies including a heavy quark-antiquark pair in the final state. These samples are generated with the POWHEG method and can be used to prepare distributions at the NLO accuracy with first radiation treated according to the parton shower approach. Information related to each event is stored in the form prescribed by the Les Houches Accords. Standard parton shower Monte Carlo programs can be used to further evolve these events, and simulate events at the hadron level, ready for almost arbitrary experimental analysis. Currently the available final states are the following: (i) $t+\bar{t}$, (ii) $t+\bar{t}+H$, (iii) $t+\bar{t}+$ jet, while the generation of several other final states is in progress.
\end{abstract}

The 2011 Europhysics Conference on High Energy Physics-HEP 2011,

July 21-27, 2011

Grenoble, Rhône-Alpes France

\footnotetext{
* Speaker.

${ }^{\dagger}$ This research was supported by the LHCPhenoNet network PITN-GA-2010-264564, and the TÁMOP 4.2.1./B09/1/KONV-2010-0007 project.
} 
In recent years a lot of NLO QCD calculations have been presented in the literature. Highenergy experiments, notably at the LHC, have and will be benefited by the progress in our computational ability to deal with higher order corrections in scattering amplitudes with many partons involved. In order though to get the optimum benefit and to produce predictions that can be directly compared to experimental data at the hadron level, a matching with parton showers (PS) and hadronization is ultimately inevitable.

In order to construct a generic interface between parton showers and matrix element calculations at the NLO accuracy, we have chosen to combine the HELAC-NLO [3, 4] and POWHEG

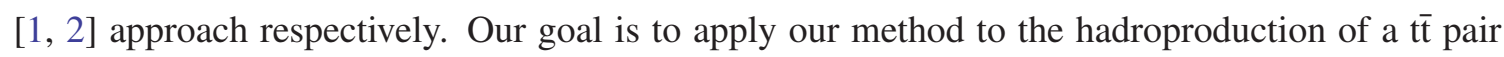
in association with Standard Model boson(s) or fermion(s) (denoted X). Due to their largest mass, the t-quarks play an outstanding role in the Standard Model and top physics is expected to yield a lot of new experimental information at the LHC (see contributions in this proceedings). The first applications of our project were the processes $p p \rightarrow \mathrm{t} \overline{\mathrm{t}}+$ jet [5] and $p p \rightarrow \mathrm{t} \overline{\mathrm{t}}+H$ [6]. In this contribution we introduce a web-page that is planned to contain event samples in the form of the Les Houches accord [7] for such processes. These events are readily usable for producing distributions at the hadron level including NLO QCD accuracy for the hard scattering process, and we expect that they will be very useful for experimental analyses.

We performed our calculations using the POWHEG-BOX [8], which requires the following ingredients: 1) flavor structures of the Born and real radiation subprocesses, 2) Born-level phase space, 3) squared matrix elements with all incoming momenta for the Born and the real-emission processes are built using amplitudes obtained from HELAC-Oneloop [9] and HELAC-PHEGAS [10], respectively. The matrix elements in the physical channels are obtained by crossing, 4) colorcorrelated squared matrix elements are taken from HELAC-Dipoles [11], 5) we use the polarization vectors to project the helicity-correlated amplitudes to Lorentz basis for writing the spincorrelated squared matrix elements. With this input POWHEG-BOX can be used to generate events at the Born level plus first radiation and store those in Les Houches Event Files (LHEF). Then one can choose any parton shower (PS) Monte Carlo program for generating events with hadrons.

In the POWHEG-BOX the first emission is the hardest one measured by transverse momentum which is can also be chosen the ordering variable in PYTHIA. If the ordering variable in the shower is different from the transverse momentum of the parton splitting, such as in HERWIG, then the hardest emission is not necessarily the first one. In such cases HERWIG discards shower evolutions (vetoed shower) with larger transverse momentum in all splitting occuring after the first emission. In addition, a truncated shower simulating wide-angle soft emission before the first emission is also needed in principle, but its effect was found small $[12,5,6]$. As there is no implementation of truncated shower in HERWIG using external LHE event files, the effect of the truncated showers is absent from our predictions.

As default, we check the consistency between real-emission, Born, color-correlated and spincorrelated matrix elements in randomly chosen phase space regions by taking the soft and collinear limits of the real-emission matrix elements in all possible kinematically degenerate channels. We compare the virtual contributions to the predictions of other programs for computing one-loop amplitudes, such as MADLOOP [13], if available. In all cases we found agreement up to 5-6 digits.

The processes we considered were also studied extensively at the NLO accuracy in the literature, which enables us to make detailed checks of our predictions. For each process we compared 

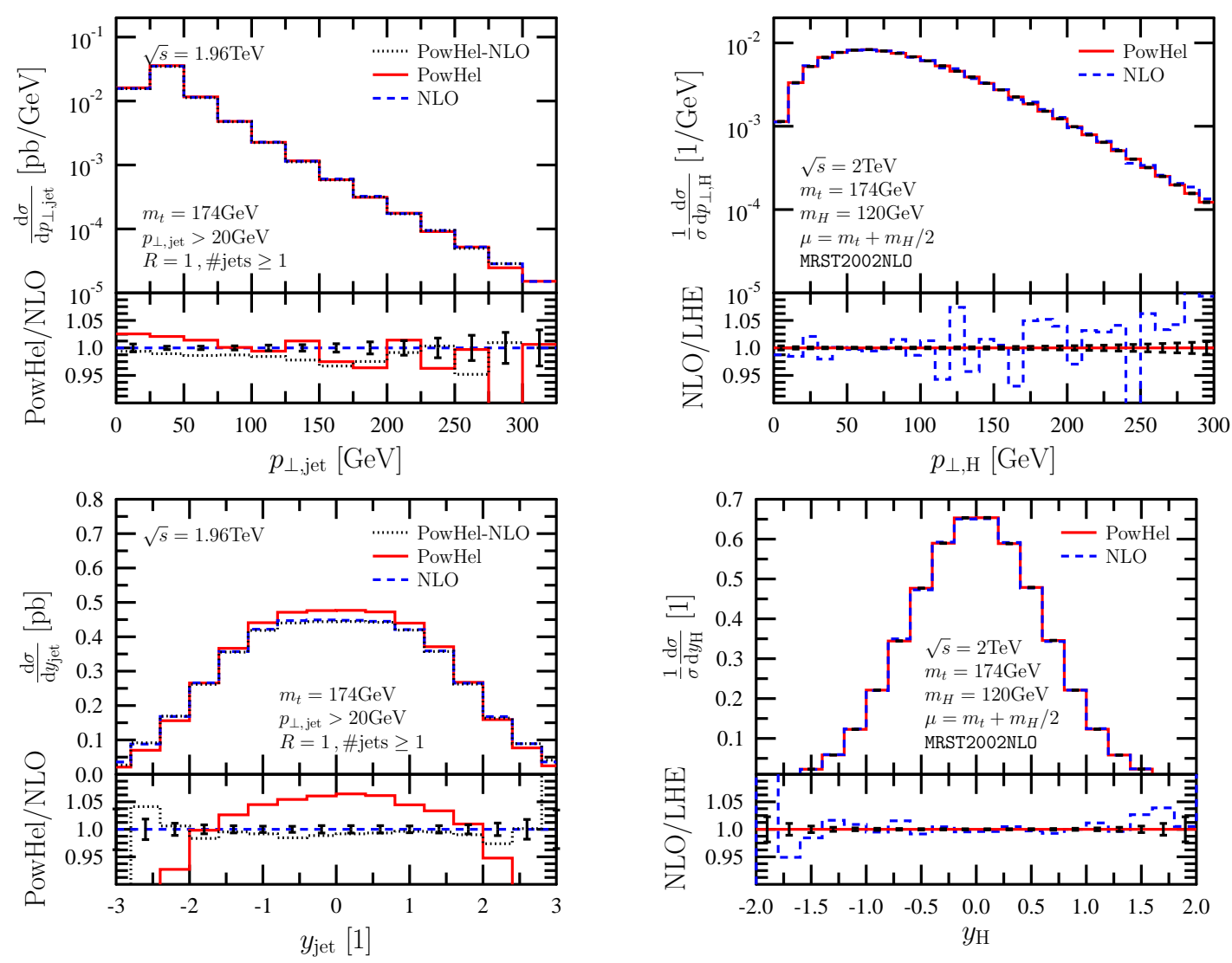

Figure 1: Distributions of transverse momemtum (upper panels) and rapidity (lower panels) of the jet in the process $p p \rightarrow \mathrm{t} \overline{\mathrm{t}}+$ jet (left) and of the Higgs particle in the process $p p \rightarrow \mathrm{t} \overline{\mathrm{t}}+H$ (right). The insets below each plot show the ratio of the predictions (PowHel/NLO).

the cross sections at the NLO accuracy to already published predictions, if available, and found agreement within the statistical uncertainty of our integrations. Finally, we also compared differential distributions based on events already including first radiation from the POWHEG-BOX with the corresponding NLO predictions. As examples, we show transverse momentum and rapidity distributions in Fig. 1 for the relevant processes. The difference between the NLO and PowHel (POWHEG+HELAC) predictions are expected to be beyond NLO accuracy [1].

Next we present selected predictions $\mathrm{t} \overline{\mathrm{t}}+X$ production with parton shower and hadronization effects at LHC. We used the event files from our webpage. Together with each set of events, one can download a sample analyis file to produce the plots presented here. In each plot we include our selection cuts, which can be altered almost freely to perform different analyses on the same events. Also the set of physical parameters used during the generation of the events can be found together with the event files. We used the last version of the PS programs, PYTHIA 6.425 [14] and HERWIG 6.520 [15], as well as for jet reconstruction, Fast Jet 2.4.3 [16].

In Fig. 2 we present distributions of the scalar sum of transverse momenta in the event, $H_{\perp}$ for the two processes $p p \rightarrow \mathrm{t} \overline{\mathrm{t}}+$ jet and $p p \rightarrow \mathrm{t} \overline{\mathrm{t}}+H$. The selection cuts implemented for these analyses are specified in Refs.[5] and [6], respectively. We can observe a softening of the spectra, 

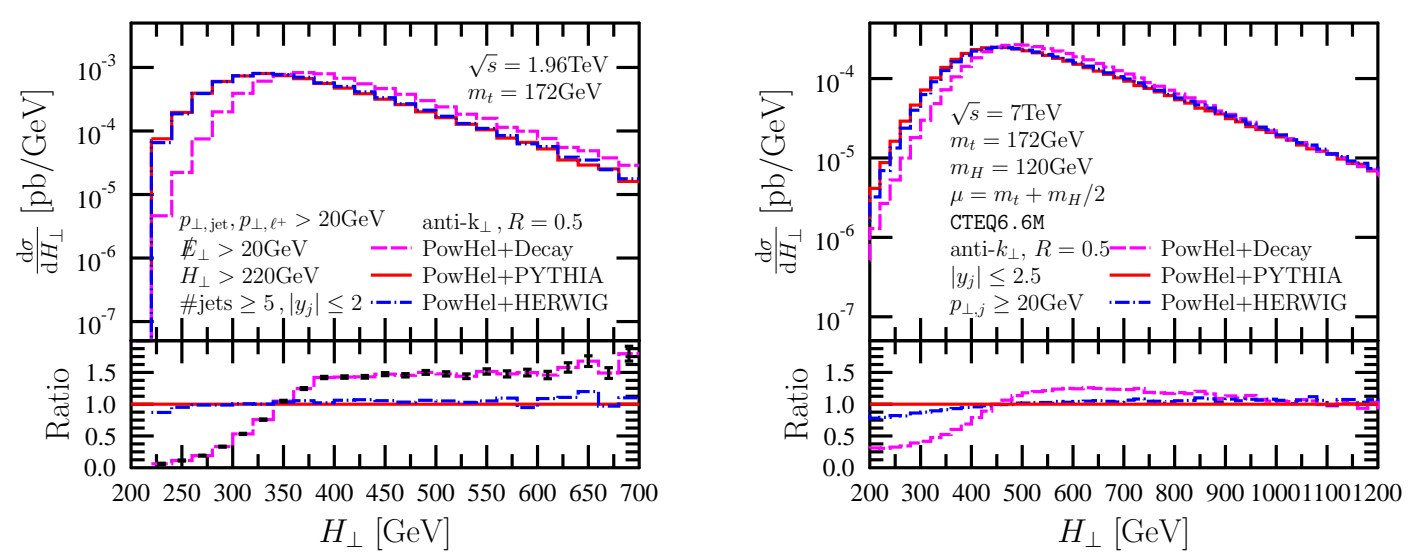

Figure 2: $H_{\perp}$-distributions of the $p p \rightarrow \mathrm{t} \overline{\mathrm{t}}+$ jet (left) and of $p p \rightarrow \mathrm{t} \overline{\mathrm{t}}+H$ (right) processes after decay of the heavy particles and after full shower Monte Carlo. The lower panels show the ratio of the predictions at the decay level to the shower MC level (POwHel+PYTHIA) and the ratio of the predictions at the hadron level using different shower MC codes (PowHel+HERWIG/PowHel+PYTHIA).

when the shower is turned on, as expected on the basis of the parton shower approach.

Interfacing NLO calculations, as structured in HELAC-NLO, with PS and hadronization effects, within the POWHEG framework, open the exciting possibility of realistic, precise and reliable simulations. We forsee large experimental and phenomenological potential of our approach. The event files produced by the POWHEG-BOX for processes $p p \rightarrow \mathrm{t} \overline{\mathrm{t}}+\mathrm{X}$, where $X$ is a hard object (SM boson, jet etc), together with the corresponding version of the program are available at http://grid.kfki.hu/twiki/bin/view/DbTheory/TthProd.

\section{References}

[1] P. Nason, JHEP 0411, 040 (2004) [arXiv:hep-ph/0409146].

[2] S. Frixione, P. Nason and C. Oleari, JHEP 0711, 070 (2007) [arXiv:0709.2092].

[3] G. Bevilacqua et al.. Nucl. Phys. Proc. Suppl. 205-206, 211 (2010) [arXiv:1007.4918].

[4] G. Bevilacqua et al., arXiv:1110.1499.

[5] A. Kardos, C.G. Papadopoulos and Z. Trocsanyi, Phys. Lett. B 705, 76 (2011) [arXiv:1101.2672].

[6] M. V. Garzelli, A. Kardos, C. G. Papadopoulos and Z. Trocsanyi, Europhys. Lett. 96, 11001 (2011) [arXiv:1108.0387].

[7] J. Alwall et al., Comput. Phys. Commun. 176, 300 (2007) [arXiv:hep-ph/0609017].

[8] S. Alioli, P. Nason, C. Oleari and E. Re, JHEP 1006, 043 (2010) [arXiv:1002.2581].

[9] A. van Hameren, C. G. Papadopoulos and R. Pittau, JHEP 0909, 106 (2009) [arXiv:0903.4665].

[10] A. Cafarella, C. G. Papadopoulos and M. Worek, Comput. Phys. Commun. 180, 1941 (2009) [arXiv:0710.2427].

[11] M. Czakon, C. G. Papadopoulos and M. Worek, JHEP 0908, 085 (2009) [arXiv:0905.0883].

[12] O. Latunde-Dada, S. Gieseke, B. Webber, JHEP 0702, 051 (2007) [arXiv:hep-ph/0612281].

[13] V. Hirschi, R. Frederix, S. Frixione, M. V. Garzelli, F. Maltoni and R. Pittau, JHEP 1105, 044 (2011) [arXiv:1103.0621].

[14] T. Sjostrand, S. Mrenna and P. Z. Skands, JHEP 0605, 026 (2006) [arXiv:hep-ph/0603175].

[15] G. Corcella et al., arXiv:hep-ph/0210213.

[16] M. Cacciari and G. P. Salam, Phys. Lett. B 641, 57 (2006) [arXiv:hep-ph/0512210], http://fastjet.fr 